\title{
A System for MR-Ultrasound Guidance during Robot-Assisted Laparoscopic Radical Prostatectomy
}

\author{
Omid Mohareri ${ }^{1,2}$, Guy Nir ${ }^{1}$, Julio Lobo ${ }^{1}$, Richard Savdie ${ }^{3}$, \\ Peter Black $^{3}$, and Septimiu Salcudean ${ }^{1}$ \\ 1 Department of Electrical and Computer Engineering, \\ University of British Columbia, Vancouver, BC, Canada \\ \{tims, omidm\}@ece.ubc.ca \\ 2 Intuitive Surgical Inc., Sunnyvale, CA \\ 3 Department of Urological Sciences, University of British Columbia, \\ Vancouver, BC, Canada
}

\begin{abstract}
We describe a new ultrasound and magnetic resonance image guidance system for robot assisted radical prostatectomy and its first use in patients. This system integrates previously developed and new components and presents to the surgeon preoperative magnetic resonance images (MRI) registered to real-time 2D ultrasound to inform the surgeon of anatomy and cancer location. At the start of surgery, a trans-rectal ultrasound (TRUS) is manually positioned for prostate imaging using a standard brachytherapy stepper. When the anterior prostate surface is exposed, the TRUS, which can be rotated under computer control, is registered to one of the da Vinci patient-side manipulators by recognizing the tip of the da Vinci instrument at multiple locations on the tissue surface. A 3D TRUS volume is then taken, which is segmented semi-automatically. A segmentation-based, biomechanically regularized deformable registration algorithm is used to register the 3D TRUS image to preoperatively acquired and annotated T2-weighted images, which are deformed to the patient. MRI and TRUS images can then be pointed at and examined by the surgeon at the da Vinci console. We outline the approaches used and present our experience with the system in the first two patients. While this work is preliminary, the feasibility of fused MRI and TRUS during radical prostatectomy has not been demonstrated before. Given the significant rates of positive surgical margins still reported in the literature, such a system has potentially significant clinical benefits.
\end{abstract}

\section{Introduction}

A standard treatment of prostate cancer is radical prostatectomy (RP), or the surgical removal of the prostate gland, via open, laparoscopic or robot assisted surgery. Studies seem to indicate that robot-assisted laparoscopic RP (RALRP) has the best outcomes, yet the rates of positive surgical margins (cancer left behind after surgery) still range between $9 \%$ and $30 \%$, depending on the center 
1]. The goal of the surgery is to remove the entire prostate and the cancer within it and extending from it, while attempting to spare as much as possible the adjacent critical structures responsible for continence (sphincter muscle) and potency (neurovascular bundles (NVB) and the cavernosal nerves). The main reason why the best trade-off between achieving oncological success (cancer removal) and functional success (continence and potency) cannot be achieved is the inability to localize, intra-opratively, the location and extent of cancer. Advances in MRI may provide spatially localized information to fill this void and aid surgical planning [2]. The combination of conventional anatomical MRI with functional (diffusion-weighted (DW) and dynamic contrast-enhanced (DCE)) MRI, known as multi-parametric MRI (mp-MRI), is emerging as an accurate tool for identifying clinically relevant tumors [3. mp-MRI is becoming an integral diagnostic tool in preoperative planning of RALRP, specifically for prediction of pathologic stage in extracapsular extension (ECE) and NVB invasion. This capability of mp-MRI to generate the most accurate characterization of prostate cancer 4], has led to the development of methods for MRI-guided treatments, mainly biopsy and brachytherapy [5].

Direct MRI-guided methods have been reported for prostate biopsy and brachytherapy, but intraoperative MRI is still cumbersome, time consuming and resource costly. Cognitive fusion, in which the clinician estimates the lesion's location in the intraoperative TRUS based on a preoperative MRI, varies greatly with expertise. A more feasible approach to allow integration of MRI data in the operating room involves registration of the preoperative MRI to the intraoperative TRUS, and visualization of the corresponding images to assist the clinician during treatment. Previously, such an approach was successfully demonstrated for prostate biopsy [6]7, and for prostate brachytherapy [8]. However, there exists no report on integration of such an approach for real-time surgical guidance using the da Vinci surgical system which is currently being used to perform more than $80 \%$ of RPs in North America.

In this work, we present a novel MR-guidance system for the da Vinci which involves an intraoperative segmentation-based MR-TRUS registration method that is integrated into a clinically used robotic TRUS imaging system, which in turn can be registered to the da Vinci system's coordinate frame. In this way a $3 \mathrm{D}$ MR volume and the preoperative surgical plan can be mapped to the da Vinci coordinate frame. The surgical instrument can then be visualized, in real-time, with respect to the preoperative $M R$ volume. In addition, since the TRUS imaging system is robotic, it can track the tip of a da Vinci surgical instrument automatically, and is registered to the preoperative MR volume, the surgical instrument itself can be used as an intuitive and easy to use control device for the surgeon to manipulate both MR and TRUS images in real-time during the procedure. The system was initially tested and validated on a prostate phantom and a data-set of six patients offline. Next, it was used with a clinical da Vinci surgical system inside a robotic operating room and tested on two patients undergoing RALRP. 


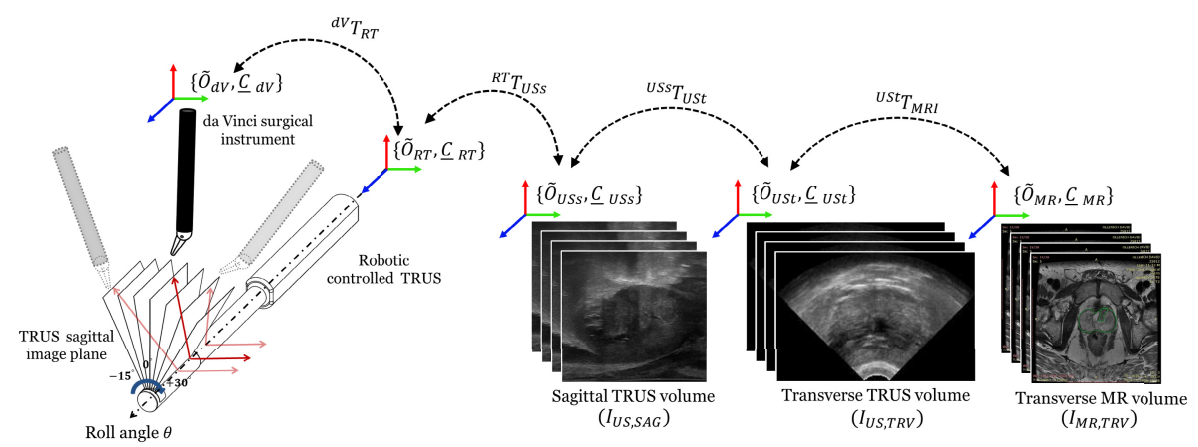

Fig. 1. A schematic of the system. da Vinci $\left\{O_{d V}, \underline{C}_{d V}\right\}$ and Robotic-TRUS $\left\{O_{R T}, \underline{C}_{R T}\right\}$ coordinate frames are registered in order to enable automatic tracking of the instrument with ultrasound. Images are then converted from cylindrical coordinates to cartesian spatial coordinates for MR-TRUS registration.

\section{Materials and Methods}

The components of our image-guided system are illustrated in Figure 1 and Figure 3. The system comprises: (i) an ultrasound system with a motorized TRUS transducer mounted on a brachytherapy setup, (ii) an external PC for image registration and display on the da Vinci console, and (iii) registration and tracking software. These components will be described next.

\subsection{TRUS Imaging System}

A previously designed and clinically used robotic TRUS manipulator was used for automatic and remote rotation (angle range \pm 45 degrees) of the TRUS transducer during the procedure 9. The sagittal TRUS imaging plane is automatically repositioned using the robot, so that the 2D ultrasound image continuously contains the tip of a specified da Vinci surgical manipulator. Automatic instrument tracking is achieved by means of a rapid and clinically feasible intraoperative registration technique that solves for the rigid homogeneous transformation between the da Vinci and the TRUS robot coordinate systems (Figure 1). The registration involves defining points on tissue surface using the da Vinci instrument tip and accurate and automatic localization of the points in both da Vinci and Robotic TRUS coordinate frames [10. This tracking method allows the surgeon at the console to control the TRUS imaging plane automatically with the tip of a da Vinci surgical instrument with an accuracy of less than $2 \mathrm{~mm}$ [9]. A BK ultrasound machine (BK Medical, Herlev, Denmark) with a 8848 4-12 MHz biplane transducer was used for imaging the prostate. Raw in phase quadrature (IQ) data was captured at $43.07 \mathrm{~Hz}$ sampling rate and saved into an external PC through a DALSA Xcelera-CL PX4 Full frame grabber card (Teledyne DALSA, Waterloo, ON). The TRUS robot was used for 3D TRUS volume acquisition by 
automatically controlling the rotation angle of the TRUS transducer and saving the location information of each image. All TRUS volumes were captured using the 214-element $6 \mathrm{~cm}$ long sagittal array with a transmit frequency of $9 \mathrm{MHz}$ and an imaging depth of $5.6 \mathrm{~cm}$. Volumes were obtained using a 90-degree rotary sweep about the probe axis with images acquired at increments of 0.2 degrees. The image capture time was 45 seconds per volume.

\subsection{TRUS-MR Registration}

The prostate gland on each transverse slice in the preoperative T2-weighted MR volume is segmented manually by a radiologist before surgery. After interpolating the intraoperative TRUS B-mode images into a 3D grid in order to obtain transverse slices from the sagittal volume, we employed a real-time semi-automatic algorithm for 3-D segmentation of the prostate in the ultrasound volume. This algorithm, described in [11, has been routinely employed during brachytherapy, and found to be a fast, consistent and accurate tool for the delineation of the prostate gland in 3D TRUS.

Based on the segmented surfaces of the prostate in the TRUS and MR volumes, we construct binary volumes. The two binary volumes can then be registered to each other in order to obtain a displacement map which can be applied to the MR volume. First, the MR binary volume is rigidly aligned (and scaled) to the TRUS binary volume using the principal axes transformation. Next, we deform the aligned MR volume to match the TRUS volume. The registration algorithm, based on the variational framework presented in [12, minimizes the

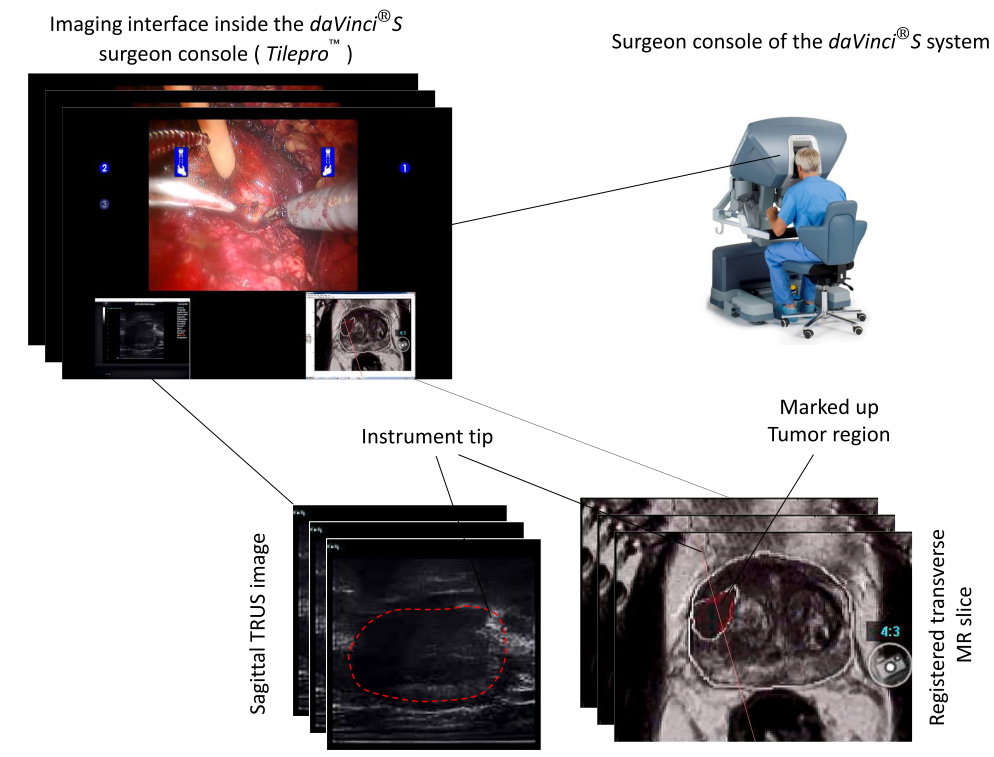

Fig. 2. Real-time imaging system interface using the da Vinci Si TilePro ${ }^{\mathrm{TM}}$ feature. 
sum of squared differences between the two binary volumes with an elastic regularization of the displacement map. The proposed method was tested offline on $n=6$ data sets of RP patients who underwent preoperative MR and intraoperative US. The MR volumes were acquired by a 3-Tesla system (Achieva 3.0T, Philips, The Netherlands) using a standard 6-channel cardiac coil with acceleration factor (SENSE) 2. In order to maintain consistent processing times, we ran the deformable registration algorithm a fixed number of 30 iterations that takes less than 60 seconds. Including the times for the semi-automated segmentation $(46 \pm 15 \mathrm{sec})$, the total run-time of the entire segmentation-based registration process is about 100 seconds. We evaluated the registration performance using the volume overlap (VO), in the sense of Dice's coefficient between the MR and the TRUS volumes after rigid and deformable registrations. The mean distance between splines on both modalities that were fitted through the center points of the urethra was also measured for the rigid and deformable registered volumes. The mean volume overlap (VO) between the registered MR and the TRUS volumes was $97.7 \pm 0.3 \%$, and the mean distance between splines fitted to the segmented urethra in the two volumes was $1.44 \pm 0.42 \mathrm{~mm}$.

\section{3 in-vivo Patient Studies and Results}

To date, two patients (ages 55 and 72; prostate specific antigen 13.5 and 28.5 $\mathrm{ng} / \mathrm{ml}$ ) with clinically organ confined prostate cancer undergoing RALRP agreed to participate in this institutional review board approved study. The main components and configuration of the system during this clinical study inside the operating room are shown in Figure 3. Both patients underwent preoperative $\mathrm{mp}-\mathrm{MR}$ and a radiologist was asked to examine their MR volume and segment the prostate and the lesions. The lesions were identified in DCE-MR images and then marked on T2-weighted images.
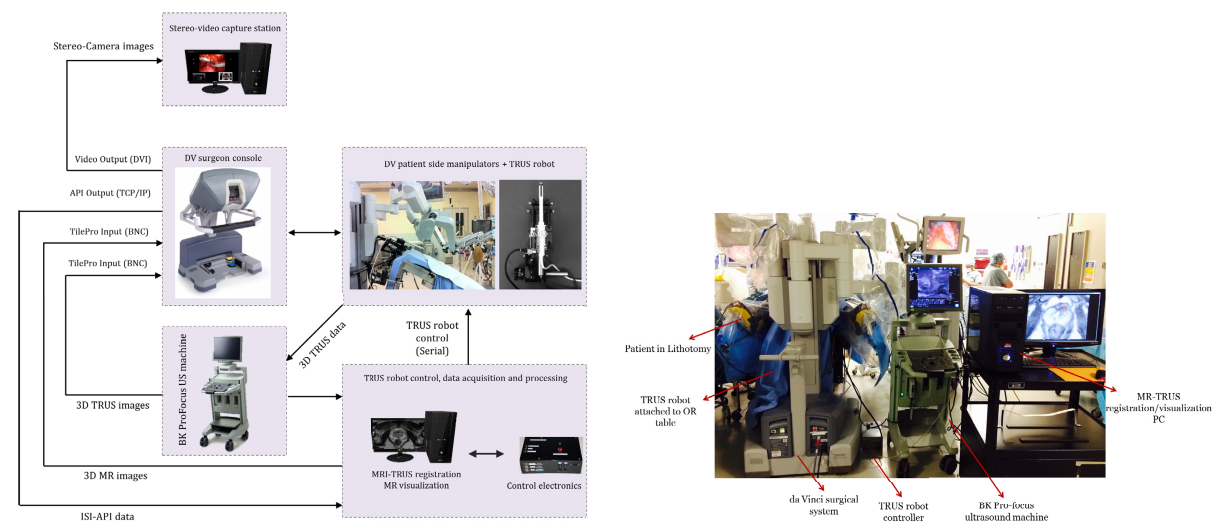

Fig. 3. The guidance system components and configuration in a da Vinci operating room is shown on the left and the operating room scenario is shown on the right. 
Table 1. Table of patient study results. $T_{r e g}$ is the duration of each registration process, $N_{r}$ is the number of times registration was repeated for that patient, $N_{f}$ is the number of fiducial points selected for instrument registration, and $T_{\text {TilePro }}$ is the total duration of TilePro usage during each case.

\begin{tabular}{|c|c|c|c|c|c|c|c|}
\hline & \multicolumn{4}{|c|}{ MR-TRUS reg. } & \multicolumn{2}{|c|}{ TRUS-dV reg. } & \multirow{2}{*}{$T_{\text {TilePro }}(\min )$} \\
\hline & $\mathrm{VO}(\%)$ & $e_{\text {reg }}(\mathrm{mm})$ & $T_{\text {reg }}(\mathrm{s})$ & $N_{r}$ & $\overline{N_{f}}$ & $T_{\text {reg }}(\mathrm{s})$ & \\
\hline Patie & $91.1 \pm 0.1$ & $2.1 \pm 0.3$ & 100 & 4 & 4 & 96 & 16 \\
\hline patient 2 & $93.0 \pm 0.3$ & $2.5 \pm 0.1$ & 100 & 5 & 4 & 110 & 28 \\
\hline
\end{tabular}

Once the patient is placed on the operating table and before docking the da Vinci robot, we attached the Robotic-TRUS system to the foot of the operating table and placed the TRUS transducer to provide optimal transverse/sagittal images of the patient's prostate as performed in standard brachytherapy volume studies. After docking the da Vinci system and start of the procedure, once the anterior surface of the prostate is visible to the surgeon, we performed the TRUS to da Vinci calibration to enable automatic tracking of the surgical instrument with ultrasound. The calibration process was performed after incision of the endopelvic fascia with 4 instrument tip locations spread across both sides of the dorsal prostate and could be completed in approximately 2 minutes. Before activating the automatic instrument tracking control mode, we acquired 3D TRUS volumes (45 seconds each) to be used by the MR-TRUS registration algorithm. The TRUS volume used for the registration was acquired immediately before the part of the procedure when the surgeon wanted to use MR images to localize tumors. This was done to ensure that the MR volume was deformed to the most current patient anatomy making the display as intuitive as possible.

After the tracking was activated, the surgeon could examine the prostate anatomy and tumor locations by moving the registered surgical instrument around, placing it on the tissue surface in the area of interest and localizing the instrument tip with respect to anatomy seen in real-time sagittal TRUS images. The corresponding transverse MR slice is also displayed based on the position of the tool tip. As it is shown in Figure 2, both real-time TRUS and the corresponding MR images are sent to the da Vinci console and displayed to the surgeon using the TilePro ${ }^{\mathrm{TM}}$ feature of the da Vinci system. The surgical view is divided into three tiles with adjustable sizes, one for the endoscopic view, one for the real-time TRUS and one for the corresponding deformed MR slice. Our graphical user interface for MR imaging display (shown in Figure 22) shows instrument tip location in the cylindrical coordinates of the TRUS system (roll angle) with a superimposed red cursor line on the $2 \mathrm{D}$ deformed MR slice. This cursor line was used by the surgeon for localization of the registered surgical instrument with respect to the segmented and annotated lesions displayed in MR images. Snapshots of the surgeon console images at the stages when the MR-guidance system was being used to localize tumors are shown in Figure 4 A summary of the registration results for both patients is listed in Table 1. 

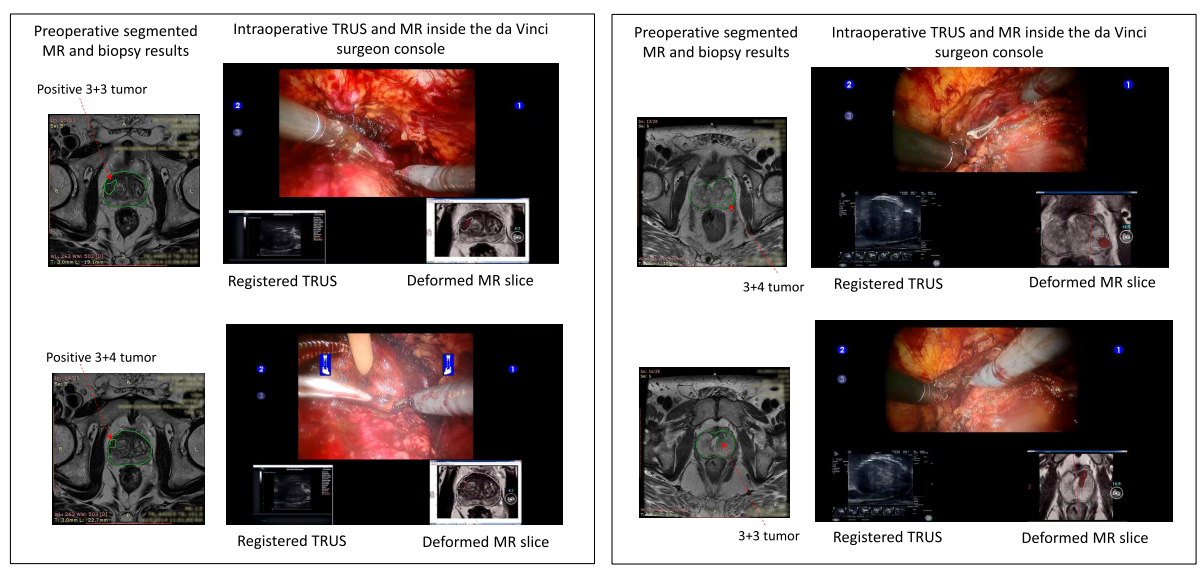

Fig. 4. Clinical study results. Both deformed MR and real-time TRUS are shown to the surgeon at the console along with the surgical endoscopic imaging for both cases.

\section{Discussions and Conclusions}

The performed surgical procedures were the first in which a surgeon was able to see registered MR images intraoperatively, and to refine the surgical planes accordingly for achieving a better surgical margin and functional outcome. During the first case, a lesion on the right side, stretching from the mid-gland anteriorly and superiorly was seen on the MR imaging system (shown in Figure 4). Based on this information, the surgeon attempted to leave as much of the NVB intact on the left "high" or more anterior dissection approach in order to get closer to the prostate. On the right, the surgeon attempted to get close to the prostate only posteriorly. The surgeon decided to perform a bilateral nerve sparing but more conservatively because of the anterior lesion on the right seen in MR images. If the lesion was posterior, then he would not have done nerve sparing on that side. During the second case, a lesion on the left posterior side was seen on the MR images and the surgeon avoided sparing the nerve on that side. The intraoperative MR imaging allows surgeons to fine tune the procedure based on the anatomy. Without MRI, they would just have a certain number and proportion of cores involved on one side or the other, but no other information on location - hence, relying mainly on intuition. Given the controversy on the "best" surgical approach in RP, as reflected in the literature, this MR-guidance could be a powerful tool to trade off positive surgical margins against potency.

Intraoperative TRUS-da Vinci and TRUS-MR registrations were performed successfully in two patients. The imaging was examined at the anterior surface of the prostate after the surgeon placed the dorsal venus complex (DVC) suture at the prostate apex, before bladder-neck dissection for localization of the tumor, and for finding the best surgical plane to achieve both better oncological and functional outcomes before performing NVB release. TRUS volume acquisition and MR-TRUS registration processes were redone (145 seconds acquisition and 
processing time) before any stage of the surgery when the surgeon wanted to use the system. An updated TRUS volume should be acquired as necessary to deform the preoperative MR images based on the current state of the prostate.

It is important for the surgeon to have intuitive control over the intraoperative imaging system in the surgical robot coordinate space. Previous work showed that surgeon-assistant coordination is inefficient. Other methods to interact with the imaging system would be adding control devices to the da Vinci console, such as another foot-pedal or hand controller or using the da Vinci masters as pointing devices [13. A major advantage of our system is that the surgeon can seamlessly use the native da Vinci input controls to move through the 3D volume of both TRUS and MRI and manipulate the 3D data.

\section{References}

1. Ficarraa, V., et al.: Systematic review of methods for reporting combined outcomes after radical prostatectomy and proposal of a novel system: The survival, continence, and potency (scp) classification. European Urology 61(3), 541-548 (2012)

2. Tan, N., et al.: Radical prostatectomy: value of prostate MRI in surgical planning. Abdominal Imaging 37, 664-674 (2012)

3. Johnson, L.M., et al.: Multiparametric MRI in prostate cancer management. Nature Reviews Clinical Oncology 11, 346-353 (2014)

4. Kozlowski, P., et al.: Combined prostate diffusion tensor imaging and dynamic contrast enhanced MRI at 3T-quantitative correlation with biopsy. Magnetic Resonance Imaging 28(5), 621-628 (2010)

5. Tempany, C., et al.: MR-guided prostate interventions. Magnetic Resonance Imaging 27(2), 356-367 (2008)

6. Hadaschik, B.A., et al.: A novel stereotactic prostate biopsy system integrating pre-interventional magnetic resonance imaging and live ultrasound fusion. Journal of Urology 186(6), 2214-2220 (2011)

7. Ukimura, O., et al.: 3D elastic registration system of prostate biopsy location by real-time 3D transrectal ultrasound guidance with MR/TRUS image fusion. The Journal of Urology 187(3), 1080-1086 (2012)

8. Reynier, C., et al.: MRI/TRUS data fusion for prostate brachytherapy: preliminary results. Medical Physics 31(6), 1568-1575 (2004)

9. Mohareri, O., et al.: Intraoperative registered transrectal ultrasound guidance for robot-assisted laparoscopic radical prostatectomy. The Journal of urology 193(1), 302-312 (2015)

10. Yip, M.C., Adebar, T.K., Rohling, R.N., Salcudean, S.E., Nguan, C.Y.: 3D ultrasound to stereoscopic camera registration through an air-tissue boundary. In: Jiang, T., Navab, N., Pluim, J.P.W., Viergever, M.A. (eds.) MICCAI 2010, Part II. LNCS, vol. 6362, pp. 626-634. Springer, Heidelberg (2010)

11. Mahdavi, S.S., et al.: Semi-automatic segmentation for prostate interventions. Medical Image Analysis 15(2), 226-237 (2011)

12. Modersitzki, J.: Numerical methods for image registration. Oxford University Press (2004)

13. Leven, J., et al.: DaVinci canvas: A telerobotic surgical system with integrated, robot-assisted, laparoscopic ultrasound capability. In: Duncan, J.S., Gerig, G. (eds.) MICCAI 2005. LNCS, vol. 3749, pp. 811-818. Springer, Heidelberg (2005) 